\title{
Viscosity of a binary mixture: Approach to the hydrodynamic limit
}

\author{
Mathieu G. McPhie, * Peter J. Daivis, and Ian K. Snook \\ Applied Physics, School of Applied Sciences, \\ RMIT University, GPO Box $2476 \mathrm{~V}$, \\ Melbourne, Victoria 3001, Australia
}

(Dated: May 18, 2006)

\begin{abstract}
We have used equilibrium and non-equilibrium molecular dynamics simulations to study the solute self diffusion coefficient and the shear rate dependence of the solution viscosity in solutions of suspended particles that range in mass ratio from $\mu=1$ up to $\mu=50$ and size ratio from $s=1 \mathrm{up}$ to $s=4.03$ at various concentrations. The zero shear rate viscosities and the initial rates of shear thinning were determined from data in the shear rate region in which the suspension is strongly shear thinning while the solvent remains Newtonian or is weakly shear thinning. The rate of shear thinning increased dramatically with solute volume fraction, regardless of whether the increase was due to increasing solute size, or increasing the solute concentration. In a series of simulations in which the mass ratio was varied while keeping the size ratio fixed at $s=1$, we found that the approach of the viscosities and self diffusion coefficients to their limiting mass ratio independent values was well described by a rather simple exponential dependence on mass ratio. The limiting infinite mass ratio values of the self diffusion coefficients and zero shear rate viscosities were plotted against solute volume fraction, and used to compute the hydrodynamic radius $R_{H}$ of the solute particles by various methods. The values of $R_{H}$ that were obtained by the different methods were reasonably consistent with each other, and indicated that the radius at which the slip boundary condition holds is slightly smaller than the cross-interaction radius between the solute and solvent particles.
\end{abstract}

\footnotetext{
${ }^{*}$ Currently at the Institut für Festkörperforschung, Teilinstitut Weiche Materie, Forschungszentrum Jülich, D-52425 Jülich, Germany; Electronic address: m.mcphie@fz-juelich.de
} 


\section{INTRODUCTION}

The self-diffusion coefficient $D$ for a single, rigid, spherical solute particle of mass $m_{2}$ and radius $a$ that are large compared to the mass and radius of the particles comprising the infinite bath of equilibrium solvent of viscosity $\eta_{s}$ in which it is dispersed, is given by the Stokes-Einstein formula [1],

$$
D=\frac{k_{B} T}{\zeta}=\frac{k_{B} T}{C \pi \eta_{s} R_{H}}
$$

where $k_{B}$ is Boltzmann's constant, $T$ is the temperature, $\zeta$ is the friction coefficient and $C$ is a numerical constant determined by the hydrodynamic boundary condition for the solvent at the surface of the solute particle. The value of $C$ is 6 for stick and 4 for perfect slip. The Stokes-Einstein relation is expected to be valid when the solute undergoes diffusive motion through a solvent that can be treated as a purely viscous hydrodynamic continuum. These conditions are usually regarded as being satisfied in the Brownian limit, conventionally defined as the limit where the solute to solvent mass ratio, $\mu=m_{2} / m_{1}$ approaches infinity [2]. Eq. (1) shows that under these conditions, the self diffusion coefficient is expected to be independent of the mass of the solute particle.

Despite its origins in Brownian motion theory, the Stokes-Einstein equation is often used to describe the relationship between the solute diffusion coefficient and size and the solvent viscosity for solutions of molecules that are of a size and mass comparable to that of the solvent, even extending to situations where the solute is just a tagged solvent molecule. However, the interpretation of the boundary condition and the hydrodynamic radius of the solute particle then become ambiguous. In an effort to clarify the circumstances under which the Stokes-Einstein equation is satisfied, several studies have recently been conducted to determine the way in which the limiting behaviour described by Eq. (1) is approached when the solute particle size and mass are increased from values that are comparable to those of the solvent $[3-7]$.

Bhattacharyya and Bagchi [3] used a mode coupling theory to investigate the diffusion coefficient of a solute particle in a solvent, where the size of the solute particle was comparable to the size of the solvent particles. They found that the Stokes-Einstein relation with the slip boundary condition is approached for size ratios of about 2-3, with only a weak dependence on mass ratio.

Nuevo, Morales and Heyes [4] used molecular dynamics simulations to investigate the size 
and mass ratio dependence of the velocity autocorrelation function and diffusion coefficient of the solute for size and mass ratios in the range 2-5 and 1-27 respectively. They found that when the density of the solute particle was much less than the density of the solvent particles, the velocity autocorrelation function oscillated strongly at short times. However, the mass ratio dependence of the self diffusion coefficient was weak in comparison to the size ratio dependence, in agreement with other studies. They used the Stokes-Einstein equation with a stick boundary condition to analyse their results, but did not discuss the validity of this boundary condition, or the value of the effective hydrodynamic radius, in detail. However, a unique feature of their work was that they also considered the concentration dependence of the solution viscosity and the solute self diffusion coefficient. They interpreted their diffusion results in terms of a truncated power series expression for the concentration dependence of the long-time self diffusion coefficient of a colloidal particle with stick boundary conditions,

$$
\frac{D(\phi)}{D}=1-k_{D, 1} \phi+\cdots
$$

where $D$ is the infinite dilution value of the solute self diffusion coefficient and $\phi$ is the volume fraction of solute. The value of $k_{D, 1}$ is 2.10 for stick boundary conditions $[8,9]$. To interpret the concentration dependence of the viscosity, they used a similar equation for the concentration dependence of the viscosity of a suspension,

$$
\frac{\eta(\phi)}{\eta_{s}}=1+k_{\eta, 1} \phi+\cdots
$$

where $\eta_{s}$ is the solvent viscosity and the value of $k_{\eta, 1}$ was assumed to be equal to the value of 2.5 calculated by Einstein [10], again assuming stick boundary conditions. The agreement between the simulation results and the theoretical expressions was only qualitative.

Ould-Kaddour and Levesque [5] used molecular dynamics computer simulations to investigate the validity of the Stokes-Einstein relation as the mass and size of the solute particles were varied. They concluded that for solutions in which the size ratio between the solute and solvent particle diameters is fixed at $s=d_{2} / d_{1}=0.5$, and the mass ratio is varied, the Stokes-Einstein formula with a slip boundary condition becomes valid when the mass ratio, $\mu$, is greater than 40 . For solutions of particles with mass ratio $\mu=1$ and varying size ratio, they concluded that the Stokes-Einstein formula with slip boundary conditions is valid for size ratios, $s$, greater than 4 . Combining the data for all systems that were deemed to be in the hydrodynamic limit, they obtained a value for the effective hydrodynamic radius of 
the solute of $a=\lambda \sigma_{12}^{*}$, where $\lambda=1.1 \pm 0.1$ and $\sigma_{12}^{*}$ is the cross-radius of the Lennard-Jones interaction between the solvent and solute particles.

Schmidt and Skinner [7] performed a series of very precise molecular dynamics simulations using a model for the solute particles that was designed to maintain the density at the same value as the average internal density of a solvent particle. In agreement with the results of Ould-Kaddour and Levesque [5], they found that using a hydrodynamic radius equal to the cross-radius of the Lennard-Jones interaction between the solute and solvent particles gave results that converged to the Stokes-Einstein relation with a slip boundary condition at sufficiently high values of the mass ratio $(\mu=100)$.

In all of the above studies, the slip boundary condition seems most appropriate because the interaction between the solvent and solute particles is an isotropic potential energy function with no tangential component, and the attractive interaction between solute and solvent particles was not strong enough to create a strongly adsorbed layer of solvent around each solute particle. Despite initial indications to the contrary [11], there now appears to be a consensus that the slip boundary condition is obtained in the Brownian limit for this type of solute-solvent interaction. However, there is not yet complete agreement on the way that the hydrodynamic radius should be calculated, especially for particles that are too light or small to have reached the Brownian limit. It remains to be determined whether, by a suitable choice of the hydrodynamic radius, the range of validity of the Stokes-Einstein relation could be extended to lower mass or size ratios than the limiting values that have so far been observed.

We have published the results of a preliminary investigation of the behaviour of the solute and solvent velocity autocorrelation functions, memory functions, self diffusion coefficients and suspension viscosities as a function of the mass ratio $\mu$ for particles with size ratio $s=1$ interacting via a truncated and shifted Lennard-Jones potential [6]. The viscosity of a suspension is generally more important in industrial and technological applications than the self diffusion coefficient of the solute, but apart from the investigation of Nuevo et al. [4] and our previous study, there have been no detailed studies of changes in the viscosity of a nanocolloidal suspension, as the Brownian limit is approached. Our results showed that the memory function for the solute decayed rapidly in comparison to its velocity autocorrelation function, at high mass ratio $\mu=10$ and low solute concentration. This was interpreted as a signature of the approach to the Brownian limit. The shear rate dependence of the viscosity 
became more pronounced as the mass ratio was increased, so that for the highest mass ratio $\mu=50$ studied, the solution viscosity was significantly shear thinning over a range of shear rates for which the solvent was essentially Newtonian. The concentration dependence of the solute self diffusion coefficient and the zero shear rate suspension viscosity were also studied. When analysed in terms of the thermodynamic (rather than hydrodynamic) volume fraction, values of $k_{D, 1}=0.95$ and $k_{\eta, 1}=2.33$ were found at mass ratios of $\mu=10$ and $\mu=20$ respectively. These values did not represent asymptotic high mass ratio limits, and no detailed discussion of the of hydrodynamic radius, the boundary condition or the hydrodynamic volume fraction was given.

In this paper, we extend our results for the disparate mass, $s=1$ systems, including higher mass ratio $(\mu=50)$ and concentration $\left(x_{2}=0.5\right)$ data. We determine the infinite mass ratio limiting behaviour for solute particles with $s=1$ by extrapolation. We also present results for disparate-size systems in which the volume fraction is varied by changing the concentration at fixed mass and size ratio in the first set of data, and then by changing the size and mass ratios at fixed number fraction in the second set. We use our data for the concentration dependence of the viscosity to obtain an independent measure of the hydrodynamic radius that is then compared with that obtained from the Stokes-Einstein equation assuming a slip boundary condition.

\section{SIMULATION DETAILS}

We consider two types of system. In the first, disparate-mass system, the mass ratio, $\mu$, between the solute and solvent particles is increased while keeping the particle sizes equal. In the second, disparate-size system, the solute particle size is also increased in such a way that the internal density of the solute particle approaches the average density of the solvent continuum, using a scheme that is similar to that used by Schmidt and Skinner [7]. This system is intended to more closely resemble experimental colloids where the particles are density matched to the solvent so that the effect of gravity and associated sedimentation problems are avoided.

The particles interact via a truncated and shifted Lennard-Jones potential which is modified to include a hard core [12]. A similar potential was used by Nuevo et al. [4] and by 
Schmidt and Skinner [7]. The interaction potential (in reduced units) is

$$
\phi_{a b}(r)=4\left[\left(r-c_{a b}\right)^{-12}-\left(r-c_{a b}\right)^{-6}\right]+1
$$

if $c_{a b}<r<c_{a b}+2^{1 / 6}$, where $c_{a b}$ is the core diameter of the interaction between particles of species $a$ and $b=(1,2)$. For separations $r>c_{a b}+2^{1 / 6}$, the potential is zero. The solvent (species 1) particles are taken to have no core $c_{11}=0$ and unit mass $m_{1}=1$. The solute particles (species 2) have core radius $c_{22}$ related to the size ratio and mass $m_{2}=\mu$, where $\mu$ is the mass ratio between the solute and solvent particles. The value of the cross-interaction core radius is $c_{12}=\frac{1}{2} c_{22}$. We have chosen a truncation point of $r=2^{1 / 6}$, corresponding to the minimum of the potential, which results in a hard, purely repulsive interaction (Weeks, Chandler and Andersen (WCA) [13].

To minimize the number of parameters in the model system and to best remove the influence of the nature of the interaction between the particles, all of the interaction parameters except for the core radius are taken to be the same regardless of the species of the particles.

To study the size ratio between the species, an equivalent hard sphere diameter of the particles must be approximated. Hess, et al. [14] suggest a number of methods to approximate the size of the particles in a one-component equilibrium WCA fluid. The simplest method they outline is also the one which gives the best results in their study of the comparison between a modified Carnahan-Starling relation and results obtained from simulations. In that method the diameter of the particles is that value of $r$ for which $\phi(r)=k_{B} T$. We here take the reduced temperature of the system to be $k_{B} T=1$ in which case the solvent particles have diameter $d_{1} \approx 1$ and the solute particles $d_{2} \approx 1+c_{22}$.

The systems are simulated using the homogeneous SLLOD equations of motion which induces a linear velocity profile in the fluid. The temperature and pressure of the system are controlled via a Gaussian isokinetic thermostat [15] and a modified Nosé-Hoover barostat 
devised by Melchionna, Ciccotti and Holian [16]. The full equations of motion are

$$
\begin{aligned}
\dot{\mathbf{r}}_{i} & =\frac{\mathbf{p}_{i}}{m_{i}}+\mathbf{i} \dot{\gamma} y_{i}-\zeta\left(\mathbf{r}_{i}-\mathbf{R}\right) \\
\dot{\mathbf{p}}_{i} & =\mathbf{F}_{i}-\mathbf{i} \dot{\gamma} p_{y i}-(\alpha+\zeta) \mathbf{p}_{i} \\
\alpha & =\frac{1}{2 K_{0}} \sum_{i}\left[\frac{\mathbf{p}_{i}}{m_{i}} \cdot \mathbf{F}_{i}-\dot{\gamma} \frac{p_{x i} p_{y i}}{m_{i}}\right]-\zeta \\
\dot{\zeta} & =\frac{1}{Q_{p}}\left(\frac{p}{p_{0}}-1\right) \\
\dot{V} & =3 \zeta V
\end{aligned}
$$

where $\mathbf{r}_{i}, \mathbf{p}_{i}$ and $m_{i}$ are the position, peculiar (non-streaming) momentum and mass of the $i$-th particle, $\dot{\gamma}$ is the strain rate, $\mathbf{R}$ is the position of the centre of mass, $p_{0}$ is the desired pressure, $\alpha$ is the thermostat multiplier, $K_{0}$ is the total kinetic energy, $\zeta$ is the barostat multiplier, $Q_{p}$ is a parameter associated with the strength of the barostat and $V$ is the system volume. Note that the expression for the thermostat multiplier differs from the usual Gaussian isokinetic thermostat to include the effect of the barostat. To maintain the velocity profile the SLLOD equations of motion should be used in conjunction with compatible periodic boundary conditions such as those of Lees and Edwards [17].

The instantaneous pressure is given by $p=\frac{1}{3} \operatorname{Tr}\{\mathrm{P}\}$ where $\mathrm{P}$ is the pressure tensor. The microscopic expression for the pressure tensor in a homogeneous system with only pairwise additive interactions, is [15]

$$
\mathrm{P} V=\left\langle\sum_{i} \frac{\mathbf{p}_{i} \mathbf{p}_{i}}{m_{i}}-\frac{1}{2} \sum_{i, j} \mathbf{r}_{i j} \mathbf{F}_{i j}\right\rangle,
$$

where $\mathbf{r}_{i j}=\mathbf{r}_{j}-\mathbf{r}_{i}$ is the minimum-image pair separation vector and $\mathbf{F}_{i j}$ is the pair force between particles $i$ and $j$. In systems of particles with no internal degrees of freedom interacting via a central force the instantaneous pressure tensor is symmetric.

The shear rate dependent viscosity of the suspension can be calculated by

$$
\eta(\dot{\gamma})=-\frac{\mathrm{P}_{x y}}{\dot{\gamma}}
$$

where $\mathrm{P}_{x y}$ is the $x y$ component of the pressure tensor, which is always symmetric for the simulations reported here.

Recently there has been much work on a new class of integrators [18-22]. These so-called symplectic integrators are stable over a large range of time step $(\Delta t)$ values. The well known 
velocity-Verlet integrator $[23,24]$ is an example of an equilibrium symplectic integrator but is only applicable to Newtonian equations of motion.

Zhang et al. [25, 26] have developed symplectic integrators for two sets of equations of motion which include the Gaussian thermostat. The first integrator was developed for the undriven, equilibrium equations of motion with the Gaussian isokinetic thermostat [25]. The second integrator is an extension of this to include the shear rate dependent terms of the SLLOD equations of motion including the shear rate dependent thermostat multiplier [26][Appendix B]. We have extended this series of integrators to include the barostat terms, details of which may be found in the appendix.

The Zhang series of integrators possess superior stability and exhibit much less drift than the 4th order Gear integrator [27], when applied to these equations of motion. They do however have a higher degree of error of $O\left(\Delta t^{3}\right)$. These errors do not accumulate in any particular direction in the conserved quantities so that the energy or the temperature remain constant or fluctuate around a central value. Zhang et al. [26] have also shown that numerical errors in the pressure and other non-conserved properties are less for the Zhang series of integrators than for the Gear integrator.

\section{VISCOSITY OF A DISPARATE-MASS BINARY FLUID}

The first series of investigations considers a model suspension where the mass of the solute particles is varied while keeping the size ratio constant at $s=1$. The core diameter of the solute particles is set to zero, so that the only difference between the solute and solvent particles is their respective masses. This is an interesting system from a theoretical point of view since the Brownian limit has often been taken to mean that the mass ratio between the solute and solvent particles goes to infinity, $\mu \rightarrow \infty$ [28], without referring to the size ratio.

Two sets of simulations were performed to test the mass ratio dependence of the viscosity of this model system. The simulations were carried out at a constant reduced temperature of $T=1.0$ and number density of $n=0.85$, with the total number of particles being set to $N=2048$.

The first set of simulations had mass ratios $\mu=\{1-10,20,30,40,50\}$ at a constant number of solute particles $N_{2}=80$ and total number of particles $N=2048$, giving a 
number fraction of $x_{2}=N_{2} / N=80 / 2048 \approx 0.04$ for the number fraction of solute particles. (Generally only results for the mass ratios $\mu=\{1,2,5,10,20,50\}$ are shown for clarity.) The shear rate was varied in steps of 0.04 up to 0.2 , after which it was varied in steps of 0.2 up to 1.6. The number of time steps used was 20,000 for the zero shear rate run; 120,000 for the $\dot{\gamma}=0.04$ and 0.08 runs; 80, 000 for the $\dot{\gamma}=0.12$ and 0.16 runs; and 40, 000 for the higher shear rate runs. Half of the time steps were for equilibration and the other half were for production of results.

The second set of simulations had mass ratios $\mu=\{1,2,5,10,20\}$ and number fractions $x_{2}=\{0.04,0.1,0.2,0.5\}$, corresponding to solute numbers $N_{2}=\{80,205,410,1024\}$, again keeping the total number of particles fixed at $N=2048$. The shear rate was varied in steps of 0.04 up to 0.2 . The number of time steps used in this set of simulations was the same as those used in the first set of simulations.

The viscosity computed from the first set of disparate-mass simulations is shown in Fig. 1. The viscosity shows typical shear thinning but also an interesting phenomenon where the viscosities tend to become equal regardless of mass ratio at high shear rates. The degree of shear thinning is increased in the solutions with higher mass ratios, and the onset of shear thinning occurs at a much lower shear rate for the $\mu=50$ solution than for the pure solvent $(\mu=1)$. This trend is also observed in the solutions with lower mass ratio, but is less pronounced. By symmetry arguments it can be shown that if a power series expansion of the shear rate dependence of the viscosity exists, then to leading order in $\dot{\gamma}$, the viscosity goes as the square of the shear rate,

$$
\eta(\dot{\gamma})=\eta_{0}-\eta_{2} \dot{\gamma}^{2}
$$

where $\eta_{0}$ is the zero shear rate viscosity and $\eta_{2}$ is the second order coefficient in the expansion. There has been much debate about the expected functional form of the viscosity at low shear rates [29-33] and the argument is far from resolved, however we found that the parabolic functional form described our data well, and we used it as a convenient method for extrapolating to the zero shear rate viscosity and quantifying the degree of shear thinning at low shear rates. Only results for shear rates lower than the onset of power law shear thinning were included in the fits (i.e. for $\dot{\gamma} \leq 0.2$ ). At the highest values of mass ratio and concentration, the maximum shear rate included in the fits was reduced further if the quadratic fit failed to describe the data well. The results of this analysis may be seen in 


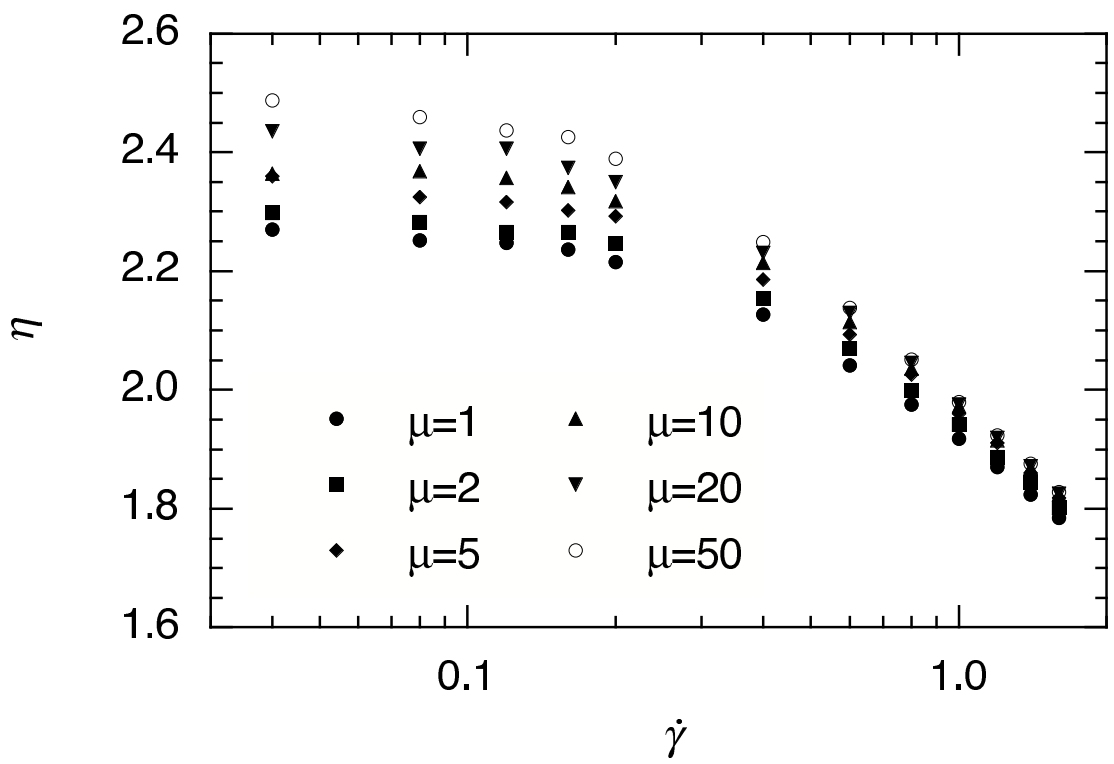

FIG. 1: Viscosity versus shear rate for different values of the mass ratio at fixed solute concentration $x_{2} \approx 0.04$ and fixed size ratio $(s=1)$. Error bars are approximately the same size as the plot symbols at low shear rates and smaller than the plot symbols at high shear rates and are omitted for clarity.

Table I. As expected, the viscosity of the suspension increases with increasing mass ratio and concentration. The first row of data $(\mu=1)$ demonstrates the reproducibility of our results. For this value of $\mu$, the solvent and solute are identical, so each number fraction represents a system of $100 \%$ solvent. The values of $\eta_{0}$ in this row are all within uncertainties of each other, and the same is true for the values of $\eta_{2}$. The zero shear rate viscosity of the pure solvent is obtained by averaging these values, giving $\eta_{s}=2.261 \pm 0.004$, and the average value of $\eta_{2}$ is equal to $1.1 \pm 0.1$.

Due to the scaling units used the viscosity of the pure solute fluid $\left(x_{2}=1\right)$ is related to the viscosity of the pure solvent by $\eta_{0}^{\text {solute }}=\mu^{1 / 2} \eta_{0}^{\text {solvent }}$. The state point of the solute fluid is the same as that of the solvent fluid, that is the temperature and pressure remain constant under this scaling.

We expect the value of the zero shear rate viscosity to become mass-independent as the mass ratio is increased at a given solute number fraction, similar to the self diffusion coefficient. Therefore, it should be possible to fit a function with an asymptote to the data to obtain a value of the limiting infinite-mass-ratio suspension viscosity at each value of the 
TABLE I: Mass-ratio dependence of the zero shear rate viscosity, $\eta_{0}$, and initial rate of shear thinning, $\eta_{2}$, for disparate-mass (equal size) suspensions, for various number fractions $x_{2}$.

\begin{tabular}{ccccccccc}
\hline \hline & \multicolumn{2}{c}{$x_{2}=0.04$} & \multicolumn{2}{c}{$x_{2}=0.10$} & \multicolumn{2}{c}{$x_{2}=0.20$} & \multicolumn{2}{c}{$x_{2}=0.50$} \\
$\mu$ & $\eta_{0}$ & $\eta_{2}$ & $\eta_{0}$ & $\eta_{2}$ & $\eta_{0}$ & $\eta_{2}$ & $\eta_{0}$ & $\eta_{2}$ \\
\hline 1 & $2.265(5)$ & $1.2(2)$ & $2.257(6)$ & $1.0(2)$ & $2.262(5)$ & $1.1(2)$ & $2.26(6)$ & $1.1(2)$ \\
2 & $2.290(5)$ & $1.1(2)$ & $2.346(6)$ & $1.3(2)$ & $2.435(6)$ & $1.7(2)$ & $2.716(7)$ & $2.5(2)$ \\
5 & $2.333(5)$ & $1.1(2)$ & $2.470(6)$ & $1.5(2)$ & $2.715(5)$ & $2.8(2)$ & $3.495(7)$ & $4.3(3)$ \\
10 & $2.375(6)$ & $1.4(2)$ & $2.580(5)$ & $2.2(2)$ & $2.950(5)$ & $3.3(2)$ & $4.38(1)$ & $12.8(9)$ \\
20 & $2.424(5)$ & $1.9(2)$ & $2.699(8)$ & $3.3(4)$ & $3.247(5)$ & $5.3(2)$ & $5.48(1)$ & $23(1)$ \\
30 & $2.444(6)$ & $2.1(2)$ & & & & & & \\
40 & $2.468(5)$ & $2.2(2)$ & & & & & & \\
50 & $2.473(5)$ & $2.1(2)$ & & & & & & \\
\hline \hline
\end{tabular}

number fraction. We found that our results were well described by the following function,

$$
\eta_{0}=\eta_{s}+\left(\eta_{\infty}-\eta_{s}\right)\left(1-e^{-a(\mu-1)}\right)
$$

where $\eta_{\infty}$ is the limiting value of the viscosity at infinite mass ratio and $\eta_{s}$ is the solvent $(\mu=1)$ viscosity. This function has the desired behaviour - at a mass ratio of $\mu=1$, we have $\eta_{0}=\eta_{s}$, and at infinite mass ratio, the suspension viscosity approaches a constant value, $\eta_{\infty}$ - but we know of no theoretical justification for this functional form for the mass ratio dependence.

The results of non-linear least squares fits to Eq. 9 are shown in normalized form in Figure 2. A surprising result is that the rate of approach to mass-independence varies nonmonotonically with concentration. The value of $a$ is smallest for number fraction $x_{2}=$ 0.04, then it increases significantly at $x_{2}=0.10$, after which it begins to decrease again. Encouraged by the success of this relatively simple description, we have reanalysed our data for the mass ratio dependence of self diffusion coefficients reported in our earlier work [6], using the functional form

$$
\frac{D-D_{\infty}}{D_{0}-D_{\infty}}=e^{-b(\mu-1)}
$$

The results shown in Fig. 3 indicate that Eq. 10 describes the data surprisingly well, and once again we observe the surprising result that the approach of the self diffusion coefficient 


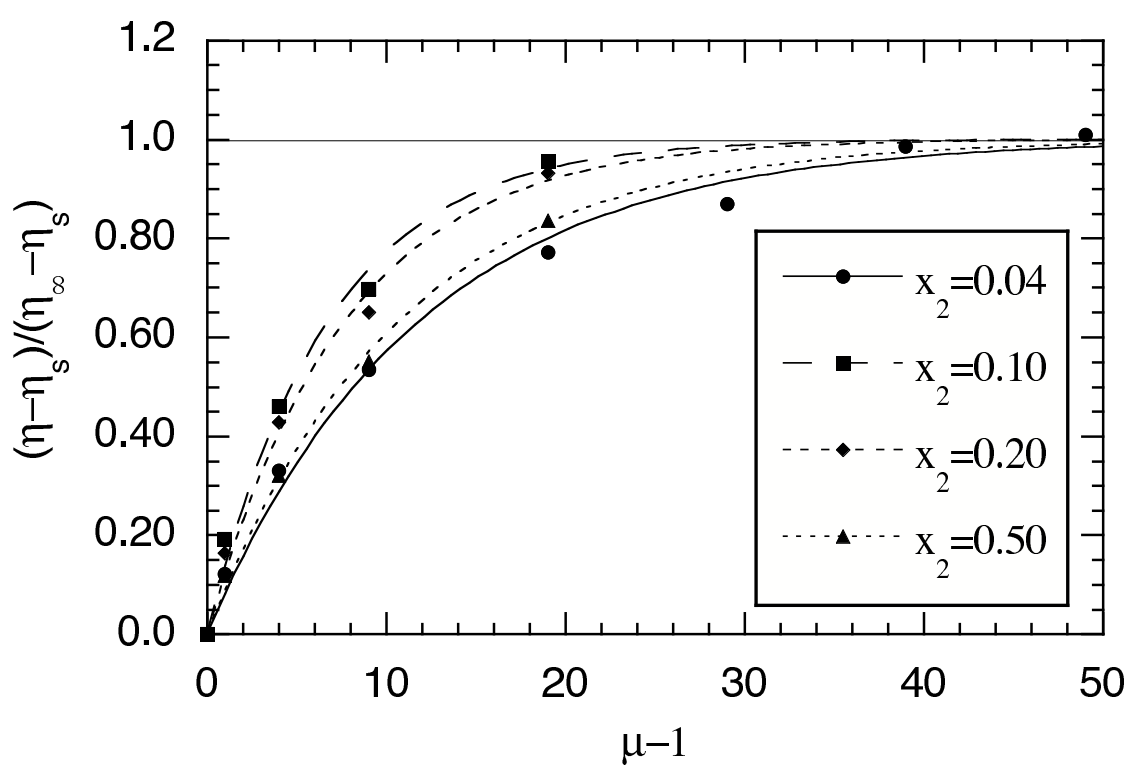

FIG. 2: Approach of the viscosity to a mass-ratio independent value for different values of the solute concentration.

to mass-independence occurs most slowly for the lowest concentration system. Values of the

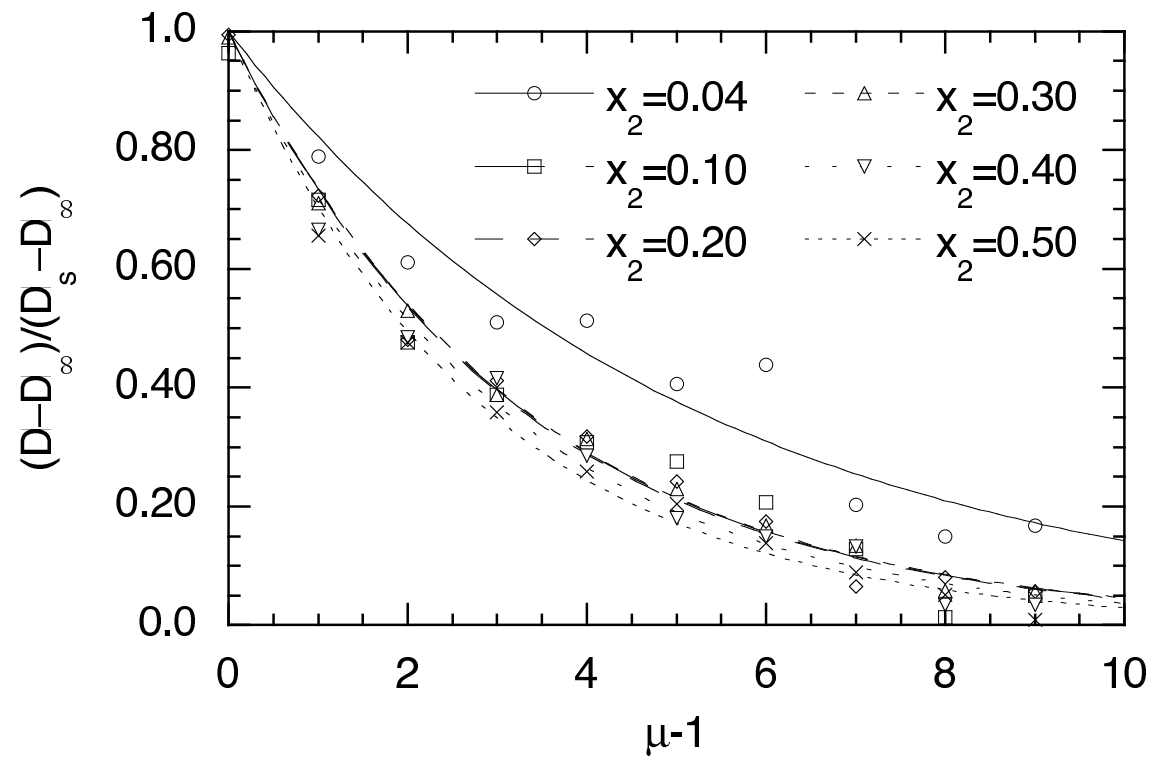

FIG. 3: Approach of the solute self diffusion coefficient to a mass-ratio independent value for different values of the solute concentration.

limiting infinite mass ratio viscosities and self diffusion coefficients, along with the rates of approach to mass ratio independence, are shown in Table II.

The concentration dependence of the self diffusion coefficient is very well fitted by a 
TABLE II: Limiting infinite mass ratio values of the solution viscosity and solute self diffusion coefficient and their rate of approach to the limit (see Eq. 9 and Eq. 10), for various number fractions $x_{2}$.

\begin{tabular}{ccccc}
\hline \hline$x_{2}$ & $\eta_{\infty}$ & $a$ & $D_{\infty}$ & $b$ \\
\hline 0.04 & $2.471(4)$ & $0.093(6)$ & $0.052(2)$ & $0.20(6)$ \\
0.1 & $2.72(3)$ & $0.15(2)$ & $0.0501(7)$ & $0.31(4)$ \\
0.2 & $3.32(7)$ & $0.13(2)$ & $0.0435(7)$ & $0.31(3)$ \\
0.3 & & $0.0381(5)$ & $0.31(2)$ \\
0.4 & & $0.034(1)$ & $0.33(3)$ \\
0.5 & $6.1(2)$ & $0.09(1)$ & $0.0287(8)$ & $0.35(2)$ \\
\hline \hline
\end{tabular}

quadratic function that gives a value for $D$ at infinite dilution and infinite mass ratio of $D_{\infty}(\phi=0)=0.056 \pm 0.001$. This result can be used, with the solvent viscosity found previously $\left(\eta_{s}=2.261 \pm 0.004\right)$ to obtain a value of $R_{H}=0.63 \pm 0.01$ for the hydrodynamic radius, assuming that the slip boundary condition applies. The cross radius, suggested by Ould-Kaddour and Levesque [5] as a possible candidate for the hydrodynamic radius, is only 0.5 , which is significantly less than this value.

We now consider the concentration dependence of the viscosity. The solution viscosity is plotted against the thermodynamic volume fraction of solute for different values of the mass ratio in Fig. 4. The concentration dependence of the limiting infinite mass ratio viscosity from Table II is also shown. The short dashed line is the expression for the viscosity of a suspension of colloidal hard spheres including hydrodynamic interactions with the stick boundary condition, given by Wajnryb and Dahler [34],

$$
\frac{\eta}{\eta_{s}}=1+2.5 \phi+5.9147 \phi^{2}
$$

and the long dashed line is their expression for slip boundary conditions,

$$
\frac{\eta}{\eta_{s}}=1+\phi+1.8954 \phi^{2}
$$

At low concentrations, the concentration dependence of the viscosity for infinite mass ratio might be expected to resemble the results for a colloidal suspension, as described by the Einstein relation, Eq. (3) with $k_{\eta, 1}=2.5$. This equation predicts that the viscosity 


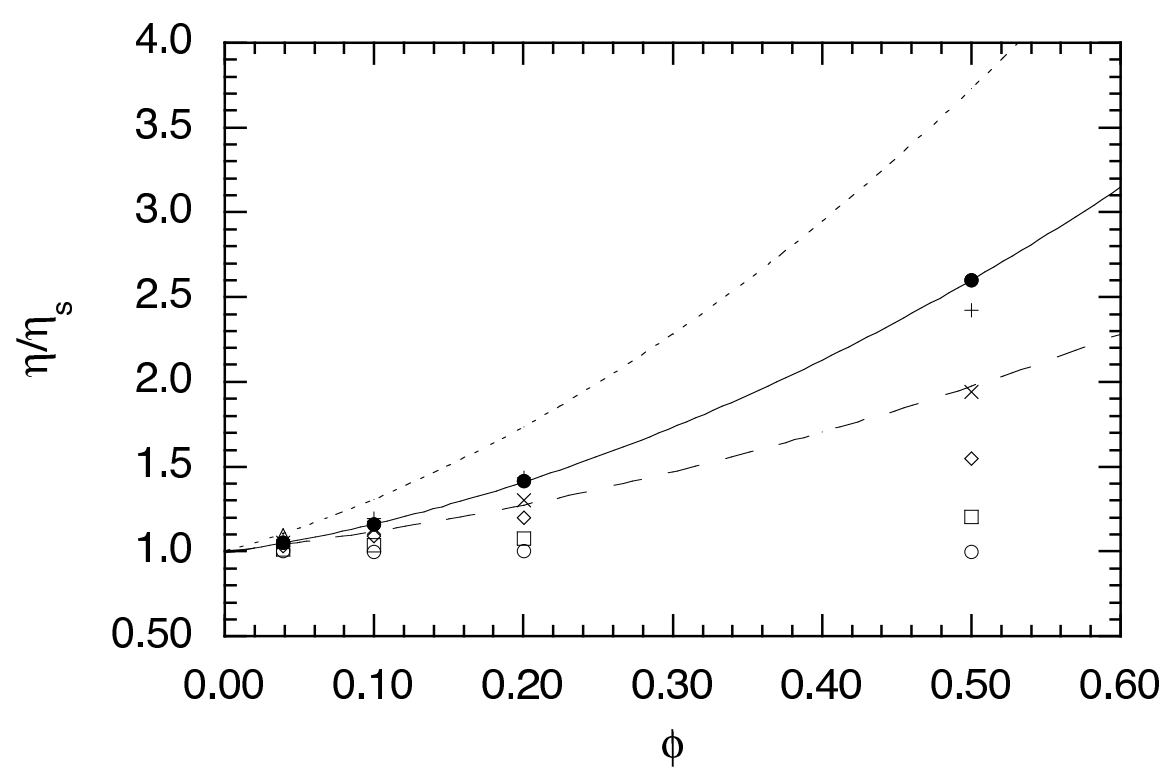

FIG. 4: Relative zero shear rate viscosity versus thermodynamic volume fraction for different solute to solvent particle mass ratios, keeping the size ratio constant at $s=1$. The mass ratios are $\mu=1$ (unfilled circles), $\mu=2$ (squares), $\mu=5$ (diamonds), $\mu=10$ (crosses) and $\mu=20$ (plus signs), and $\mu=\infty$ (filled circles). The solid line is a quadratic fit to the $\mu=\infty$ data and the short and long dashed lines are the theoretical curves for stick and slip boundary conditions respectively.

should be simply related to the viscosity of the solvent and the hydrodynamic volume fraction occupied by the solute particles, independent of the solute to solvent particle mass ratio. The Einstein expression is derived from a hydrodynamic calculation of the flow around a massive solute particle suspended in a fluid, and thus will become valid in the hydrodynamic limit. This limit is reached when the solvent particles act not as a collection of microscopic particles, but as a continuous fluid, and in this sense could also be called the continuum limit. The conditions for this limit will be satisfied when the time scale associated with the motion of the solute particles becomes much longer than the time scale associated with the relaxation of the solvent fluid. An appropriate time scale for the solute particles can be defined by the average time taken to move one particle diameter. If the solute particles are in thermal equilibrium with the solvent, this time will increase as the square root of the mass ratio, $\tau \propto \mu^{1 / 2}$. In the Brownian limit, $\mu \rightarrow \infty$, this time scale will also go to infinity $\tau \rightarrow \infty$. Therefore a sufficient condition for the approach to the hydrodynamic limit is that the Brownian limit is approached.

The results of fitting the Einstein equation to the low number fraction zero shear rate 
viscosities, up to and including $x_{2}=0.20$, were presented in a previous publication [6]. The results showed that the concentration dependence described by the Einstein formula is approached, but not reached, as the mass ratio is increased to $\mu=20$. A similar result was found by Nuevo et al. [4]. It is now clear that there are reasons for expecting that the Einstein equation will not describe the limiting behaviour of this system. We have already seen that the slip boundary condition seems to be most appropriate for the systems that we are simulating, whereas the Einstein equation assumes a stick boundary condition. It is also known $[35,36]$ that the Einstein relation is only expected to be valid for very dilute suspensions, $\phi \lesssim 0.10$. Therefore, in this paper, we have interpreted our results using Wajnryb and Dahler's extension of Einstein's formula to second order in volume fraction with stick boundary conditions (Eq. 11) and their expression for the concentration dependence of the viscosity to second order in volume fraction, assuming slip boundary conditions (Eq. 12). The coefficient of the first order term of Eq. 11 is Einstein's result 2.5, and the coefficient of the second order term, 5.9147 is an improved value of the one originally calculated by Batchelor [37] as 6.2. Eq. 12 [34] appears to be the only result published for the slip boundary condition.

In order to accurately compare our results with theoretical predictions which give the concentration in terms of volume fraction, we must find an appropriate way to calculate the hydrodynamic volume fraction. It is helpful here to clearly distinguish between the hydrodynamic and thermodynamic volume fractions. The hydrodynamic volume fraction is the volume fraction calculated from the hydrodynamic radius, which may differ from the bare or "dry" volume of the particles. Calculation of the hydrodynamic volume fraction becomes ambiguous for very small solute particles, because it requires knowledge of the radius of the surface around the solute particle at which the hydrodynamic slip or stick boundary condition is satisfied by the solvent. For a very small particle, the hydrodynamic radius may differ by a significant fraction from the bare radius of the solute particle. On the other hand, the thermodynamic volume fraction is well defined, regardless of the sizes of the components of a solution. It is defined as

$$
\phi=\frac{V_{\text {solute }}}{V}=\frac{N_{2} v_{2}}{V}=n x_{2} v_{2}
$$

where $v_{2}$ is the partial molecular volume of solute particles,

$$
v_{2}=\left(\frac{\partial V}{\partial N_{2}}\right)_{N_{1}},
$$


$n=\left(N_{1}+N_{2}\right) / V$ is the total number density of the suspension, and $x_{2}$ is the number fraction of solute particles. The total volume of the system is given by

$$
V=N_{1} v_{1}+N_{2} v_{2}
$$

Particles with identical interactions and sizes will have identical molar volumes, regardless of their mass, in an equilibrium solution. Using this fact, we find that $v_{2}=1 / n$ where $n$ is the total number density. Eq. 13 then shows that that for this series of simulations, in which the mass ratio is varied at constant size ratio $s=1$, the thermodynamic volume fraction $\phi$ of the solute particles is equal to the number fraction, $x_{2}$. Calculation of the effective particle diameter from the thermodynamic volume fraction, assuming that all particles are identical spheres occupying one molar volume each, gives $d=1.31$. This can be used to obtain a value of the hydrodynamic radius of $R_{H}=0.655$, which agrees reasonably well with the value of 0.63 found earlier from the limiting infinite mass-ratio self diffusion coefficient and the StokesEinstein relation. The partial molecular volume is an equilibrium thermodynamic property, which will be independent of the mass of the solvent particle, but the hydrodynamic radius obtained from the self diffusion coefficient and the Stokes-Einstein relation is a dynamic property that will depend on the mass ratio. Therefore, the comparison must be made cautiously, but the thermodynamic volume fraction is nevertheless a convenient measure of concentration for the comparison in Fig. 4.

A quadratic fit to the infinite mass ratio viscosity versus thermodynamic volume fraction data shown in Fig. 4 gives the result $\eta / \eta_{s}=1+1.2(3) \phi+4.0(9) \phi^{2}$. This is significantly different from the expected result for the slip boundary condition, Eq. (??)q:eta-slip. The discrepancy could be due to an inadequate estimate of the hydrodynamic radius, or an incorrect assumption regarding the hydrodynamic boundary condition.

If the hydrodynamic radius is instead calculated from the bare radius, the volume fraction calculated for the highest concentration $\left(x_{2}=0.5\right)$ is $\phi=0.22$. This would place the highest concentration points for $\mu=\infty$ and $\mu=20$ both well above the theoretical curve for the stick boundary condition. Clearly, the stick boundary condition does not apply. However, when the volume fraction is calculated from the thermodynamic volume per particle, the highest concentration point at $\phi=0.50$, as shown in Fig. 4, falls above the theoretical curve for the slip boundary condition. If it is assumed that the slip boundary condition applies, and the volume fraction is calculated using a hydrodynamic radius that has been adjusted to force 
agreement with the theroretical curve, a hydrodynamic radius of $R_{H}=0.73$ is found. This value is larger than the Stokes-Einstein radius $0.63 \pm 0.01$ and the thermodynamic radius 0.655 but smaller than the interaction cross-radius 1.0. The agreement between the values of hydrodynamic radius calculated from the concentration dependence of the viscosity, the Stokes-Einstein equation and the thermodynamic volume per particle is fair.

\section{VISCOSITY OF A DISPARATE-SIZE BINARY FLUID}

In experimental studies of colloidal systems, the solute particles generally have constant density that is independent of the size of the particle. If this density is equal to the bulk solvent density then the effects of gravity can be ignored and the colloidal particle motion is then due solely to Brownian motion, hydrodynamic drag and interactions with other solute particles. We wish to study a system that approaches this behaviour as the size ratio between the solute and solvent particles is increased.

In our simulations of solutions of disparate-sized particles, we have changed the particle size by increasing the core of the solute particles in the potential energy function, Eq. (4). The density of the solute particles is chosen to fulfil two limiting requirements. The first is that at a size ratio of $s=d_{2} / d_{1}=1$ the mass of a solute particle equals the mass of a solvent particle, so that in the equal size limit the particles are identical. The second is that in the limit as the size ratio between the species goes to infinity $(s \rightarrow \infty)$ the mass of the solute particle is such that it has an internal mass density as close as possible to the fluid density surrounding it. A convenient method of achieving this is to take the density of the solute

particle core (see Eq. (4)), of radius $\frac{1}{2} c_{22}$, to be equal to the fluid density and the density of the shell, of thickness $\frac{1}{2}$, to be equal to the internal density of a solvent particle. Figure 5 shows the approximate size ratio obtained using this scheme. Note that in calculating the core radius for specific mass ratios, the bulk number density of the solvent is taken as the zero shear rate, pure solvent $\left(\dot{\gamma}=0, x_{2}=0\right)$ number density. This is an approximation which is good at low volume fraction but becomes progressively worse as the solute volume fraction is increased. For the volume fractions studied here this approximation is adequate.

The internal density of the solvent particles, which have mass $m_{1}=1$ and diameter $d_{1}=1$, is $6 / \pi$, and the density of the pure solvent at constant pressure is the equilibrium 


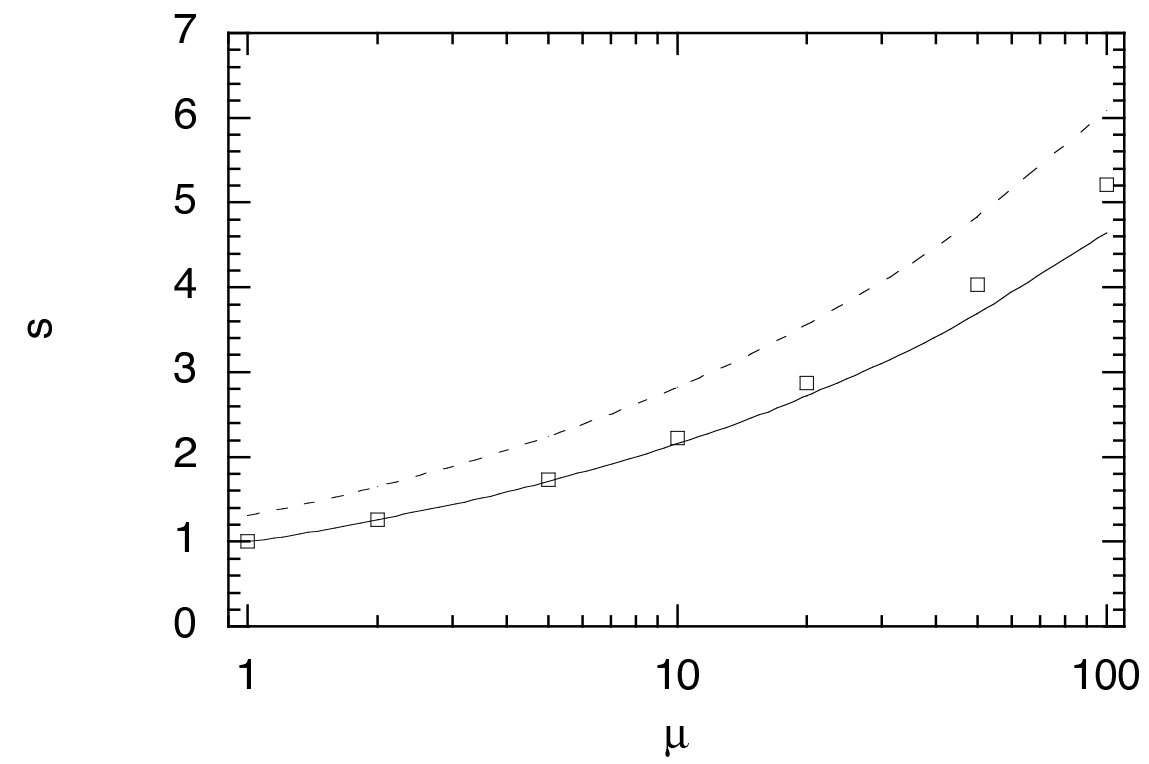

FIG. 5: Approximate size ratio $s$ between solute and solvent particles. Squares represent the composite core-shell solute particles. The solid line is the size ratio of solute particles with an internal density equal to the solvent particles and the dashed line that of neutrally buoyant solute particles.

number density $n_{0}$. The mass of the core of the solute particles is

$$
m_{\text {core }}=\frac{\pi}{6} c_{22}^{3} n_{0},
$$

and the mass of the shell is

$$
m_{\text {shell }}=\left(c_{22}+1\right)^{3}-c_{22}^{3} .
$$

It is important to be able to characterize the solute particles primarily by size. This requires that the sum of equations (16) and (17) be solved to give the core diameter $c_{22}$ as a function of solute mass. For a pure solvent number density of $n_{0}=0.85$ the core diameters corresponding to the set of mass ratios investigated may be seen in Table III.

Two sets of simulations were performed to study the effect of solute size on the viscosity of a model suspension. The simulations were performed at a constant temperature of $T=1.0$ and pressure of $p=9.04$, which corresponds to a pure solvent fluid number density of $n_{0}=0.873$. As in our previous set of simulations, the total number of particles was set to $N=2048$.

The simulations of the disparate-size mixtures were performed at constant solution pressure to ensure that the results would be comparable to our $s=1$ results in the previous 
TABLE III: Core diameters $c_{22}$, and corresponding size ratios $s$, used in the disparate-size mixture simulation, for various solute to solvent mass ratios $\mu$.

\begin{tabular}{lcc}
\hline \hline$\mu$ & $c_{22}$ & $s$ \\
\hline 1 & 0 & 1 \\
2 & 0.262 & 1.262 \\
5 & 0.735 & 1.735 \\
10 & 1.225 & 2.225 \\
20 & 1.869 & 2.869 \\
50 & 3.030 & 4.030 \\
\hline \hline
\end{tabular}

section. For equal sized particles, the thermodynamic properties of the solution remain constant as the solute to solvent particle mass ratio and the concentration are increased. In the current set, the thermodynamic properties must change when the particle size ratio or the concentration are changed. The most convenient way of maintaining comparable conditions for the two sets of simulations is to keep the solution pressure constant.

The first set of simulations was used to study the size ratio dependence of the suspension viscosity. The simulations had mass ratios $\mu=\{1,2,5,10,20,50\}$ and a constant molar concentration of solute particles of $x_{2} \approx 0.005$ corresponding to $N_{2}=10$. The volume fraction of the solute particles increases as the mass ratio increases, so the number fraction was chosen to allow the study of reasonably large size ratios, corresponding to large volume concentrations. The shear rates used were $\dot{\gamma}=\{0,0.02,0.1,0.2,0.3\}$, with a time step of either $\Delta t=0.01$ or 0.005 and a total number of time steps of 100,000 for equilibration and a further 100,000 for production of results. The $\mu=10$ and 50 simulations were extended to shear rates of $\dot{\gamma}=1.0$ in steps of 0.1 .

The second set of simulations was used to study the concentration dependence of the suspension viscosity at a fixed value of the size (and mass) ratio. The mass ratio was set at $\mu=10$, corresponding to a size ratio of $s=2.225$, and the number fraction was taken to be $x_{2}=\{0.001,0.005,0.01,0.02,0.04\}$. The shear rates, time step and numbers of time steps were the same as those used in the first set of simulations. The $x_{2}=0.005$ and 0.01 sets were extended to $\dot{\gamma}=1.0$ in steps of 0.1 .

The shear rate dependence of the viscosity for the $x_{2}=0.005$ and $\mu=10$ suspensions at 
low shear rates are shown in Figures 6 and 7. Both Figures show similar behaviour. The viscosity at low shear rates depends strongly on the number fraction and size ratio. The viscosity decreases with increasing shear rate. The suspensions with larger number fraction or larger size ratio, both of which correspond to larger volume fractions, show a greater degree of shear thinning, and this shear thinning is seen to occur at lower shear rates. For the $x_{2}=0.005, \mu=50$ suspension the Newtonian region is never reached. At large shear rates the suspension viscosities do not approach a common limiting value, unlike the $s=1$ simulations, see Figure 1. Heyes [38] has observed strong shear thinning of hard sphere

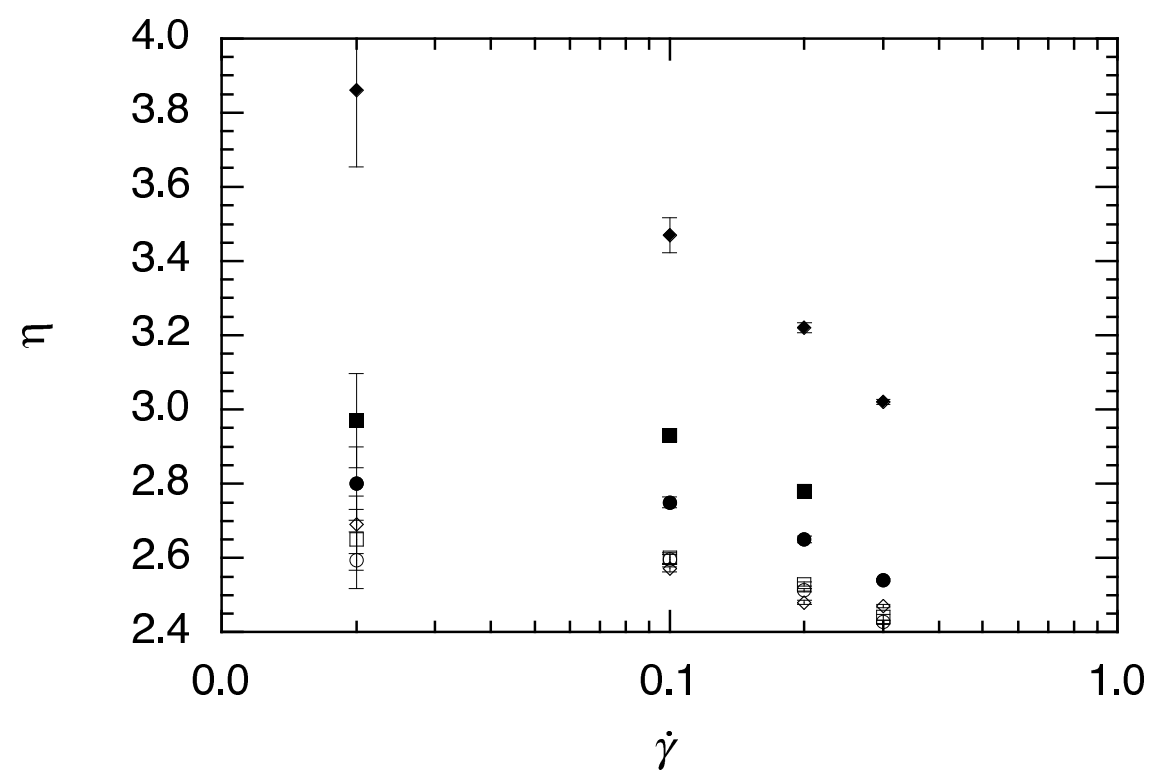

FIG. 6: Viscosity versus shear rate for the $x_{2}=0.005$ disparate-size suspension, for various mass (and size) ratios. The different symbols correspond to mass ratios of: 1 (unfilled circles), 2 (unfilled squares), 5 (unfilled diamonds), 10 (filled circles), 20 (filled squares), 50 (filled diamonds).

suspensions modelled by a Brownian dynamics simulation algorithm ${ }^{1}$ which does not include the solvent explicitly and does not give any dependence on mass ratio. Heyes found that the degree of shear thinning increased with increasing solute volume fraction, as is observed in this work.

The shear thinning behaviour of these suspensions is much more pronounced than that

\footnotetext{
${ }^{1}$ The particular Brownian dynamics algorithm used by Heyes includes the random Brownian force, the hydrodynamic drag force and the direct solute-solute interaction forces. It does not include the solvent mediated many-body hydrodynamic interactions between the solute particles.
} 


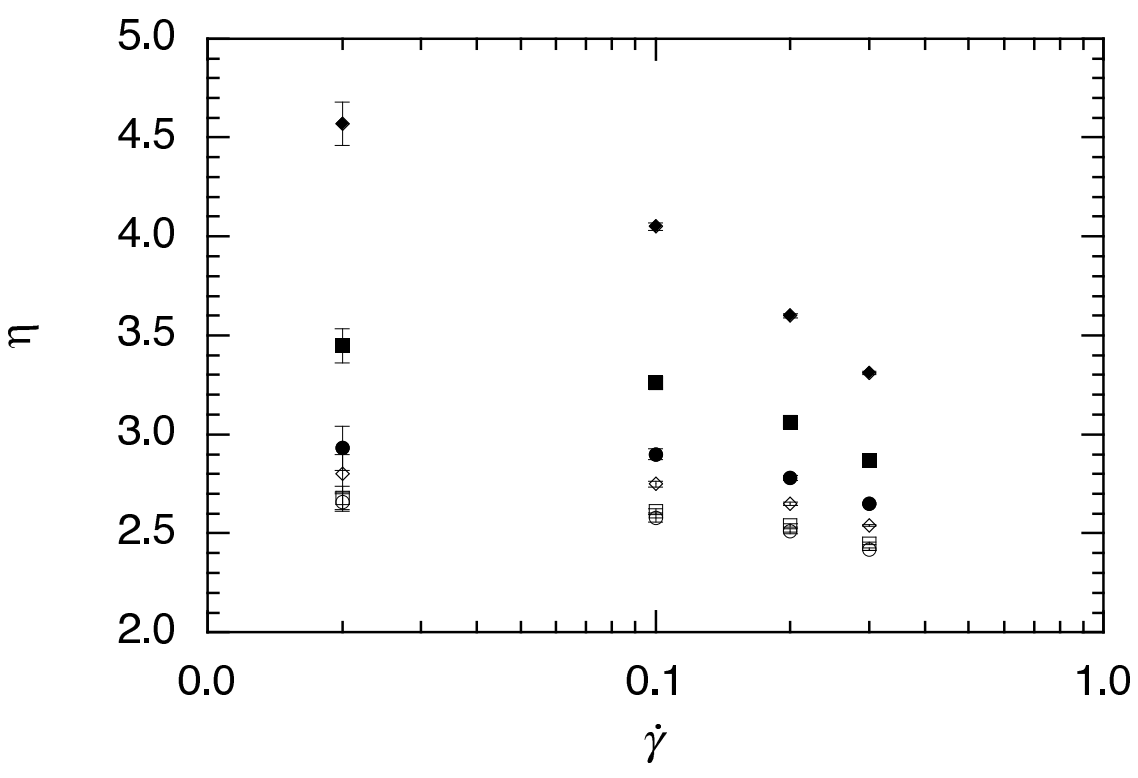

FIG. 7: Shear rate dependent viscosity of the $\mu=10(s \approx 2.225)$ disparate-size suspension, for various solute number fractions, $x_{2}=0.0$ (unfilled circles), 0.001 (unfilled squares), 0.005 (unfilled diamonds), 0.010 (filled circles), 0.020 (filled squares), 0.040 (filled diamonds).

TABLE IV: Variation of the fit coefficients in Eq. 18 with mass and size ratio at constant solute number fraction, $x_{2}=0.005$.

\begin{tabular}{ccccc}
\hline \hline$\mu$ & $s$ & $\eta_{0}$ & $\eta_{2}$ & $\eta_{4}$ \\
\hline 1 & 1 & $2.63(2)$ & $3.4(7)$ & $13(6)$ \\
2 & 1.262 & $2.63(2)$ & $2.7(8)$ & $7(7)$ \\
5 & 1.735 & $2.62(1)$ & $4.8(5)$ & $35(5)$ \\
10 & 2.225 & $2.79(2)$ & $4(1)$ & $14(8)$ \\
20 & 2.869 & $2.99(3)$ & $6.6(10)$ & $32(11)$ \\
50 & 4.03 & $3.60(7)$ & $12(3)$ & $63(20)$ \\
\hline \hline
\end{tabular}

of the equal size suspensions described in the previous section. To obtain the zero shear rate viscosities and a quantitative measure of the initial rate of shear thinning, we fitted a second order polynomial function to the viscosity versus squared shear rate data, given by

$$
\eta(\dot{\gamma})=\eta_{0}-\eta_{2} \dot{\gamma}^{2}+\eta_{4} \dot{\gamma}^{4}
$$

The results of these fits are shown in Table IV and Table V.

We again used the thermodynamic volume fraction as our measure of concentration for 
TABLE V: Variation of the fit coefficients in Eq. 18 with solute number fraction, $x_{2}$ at constant mass ratio $\mu=10$ and size ratio $s=2.225$.

\begin{tabular}{cccc}
\hline \hline$x_{2}$ & $\eta_{0}$ & $\eta_{2}$ & $\eta_{4}$ \\
\hline 0 & $2.62(3)$ & $3.4(13)$ & $12(11)$ \\
0.000976563 & $2.64(2)$ & $2.9(9)$ & $8(8)$ \\
0.004882813 & $2.79(2)$ & $4.0(10)$ & $14(8)$ \\
0.009765625 & $2.95(4)$ & $4.8(15)$ & $17(13)$ \\
0.01953125 & $3.36(4)$ & $9.2(15)$ & $42(13)$ \\
0.0390625 & $4.27(3)$ & $21.6(12)$ & $120(10)$ \\
\hline \hline
\end{tabular}

the disparate-size suspensions. In constant pressure simulations at low solute concentration, it is a very good approximation to assume that the partial molecular volume of the solvent remains constant regardless of solute concentration. Then, if $n_{0}$ denotes the number density in the pure solvent, the partial volume of the solute particles in a suspension at the same pressure will be given by

$$
v_{2}=\frac{1}{n x_{2}}\left\{1-\frac{n}{n_{0}}\left(1-x_{2}\right)\right\}
$$

so that the volume fraction of the solute particles will be given by

$$
\phi=1-\frac{n}{n_{0}}\left(1-x_{2}\right)
$$

The partial volume of the solute particles in the first set of simulations, in which the solute number fraction remains fixed at $x_{2} \approx 0.005$, was calculated using equations Eq. 14 and Eq. 19. The first expression was evaluated by performing simulations at the state points $\left(N=2047, N_{2}=9\right)$ and $\left(N=2049, N_{2}=11\right)$, with $T=1.0, p=9.04$ and $\dot{\gamma}=0$, and approximating $v_{2}$ by

$$
v_{2}\left(N=2048, N_{2}=10\right)=\left(\frac{\partial V}{\partial N_{2}}\right)_{N_{1}} \approx \frac{1}{2}\left[V_{\left(N=2049, N_{2}=11\right)}-V_{\left(N=2047, N_{2}=9\right)}\right] .
$$

The partial volumes from this calculation and those calculated from Equation (19) are compared in Table VI against the bare volume of a solute particle, $\pi s^{3} / 6$, which is expected to become a good approximation to the partial volume for large values of $s$. It can be seen that the thermodynamic partial volume is very well approximated by assuming that partial volume of the solute particles remains constant. The thermodynamic volume fractions for 
TABLE VI: Partial volumes $v_{2}$ disparate-size mixture, with solute number fraction $x_{2} \approx 0.005$, for various mass ratios. Columns 4 and 5 are calculated using Equations (21) and (19), while the last column is the bare volume of a single spherical solute particle.

\begin{tabular}{llllll}
\hline \hline$\mu$ & $s$ & $n$ & & $v_{2}$ \\
\hline 1 & 1 & 0.873 & 1.145 & 1.145 & 0.524 \\
2 & 1.262 & 0.870 & 1.983 & 1.955 & 1.052 \\
5 & 1.735 & 0.862 & 4.226 & 4.273 & 2.735 \\
10 & 2.225 & 0.848 & 8.140 & 8.117 & 5.768 \\
20 & 2.869 & 0.821 & 16.013 & 16.140 & 12.365 \\
50 & 4.030 & 0.745 & 41.240 & 41.326 & 34.270 \\
\hline \hline
\end{tabular}

TABLE VII: Solute volume fractions in the disparate-size mixtures calculated from Eq. (19) for the $x_{2}=0.005$ constant solute number fraction disparate-size mixtures at various mass ratios (first 3 columns) and for the $\mu=10$ constant mass ratio $(\mathrm{s} \approx 2.225)$ mixtures at various number fractions (last 3 columns).

\begin{tabular}{lcccccc}
\hline \hline & Const. $x_{2}=0.005$ & & & \multicolumn{3}{c}{ Const. $\mu=10$} \\
\cline { 5 - 6 }$\mu$ & $n$ & $\phi$ & & & $x_{2}$ & $n$ \\
\cline { 5 - 6 } 1 & 0.873 & 0 & 0 & 0.873 & 0 \\
2 & 0.870 & 0.008 & 0.001 & 0.868 & 0.007 \\
5 & 0.862 & 0.018 & 0.005 & 0.848 & 0.033 \\
10 & 0.848 & 0.033 & 0.01 & 0.824 & 0.066 \\
20 & 0.821 & 0.064 & 0.02 & 0.780 & 0.124 \\
50 & 0.746 & 0.150 & 0.04 & 0.705 & 0.225 \\
\hline \hline
\end{tabular}

the disparate-size solutions calculated using the partial molecular volumes given in column 5 of Table VI are shown in Table VII.

The zero shear rate viscosities from both sets of simulations are plotted against thermodynamic volume fraction in Fig. 8. The difference between altering the volume fraction by changing the concentration or the size of the solute particle is apparently small for these solutions. A quadratic fit to the viscosity versus thermodynamic volume fraction results for 


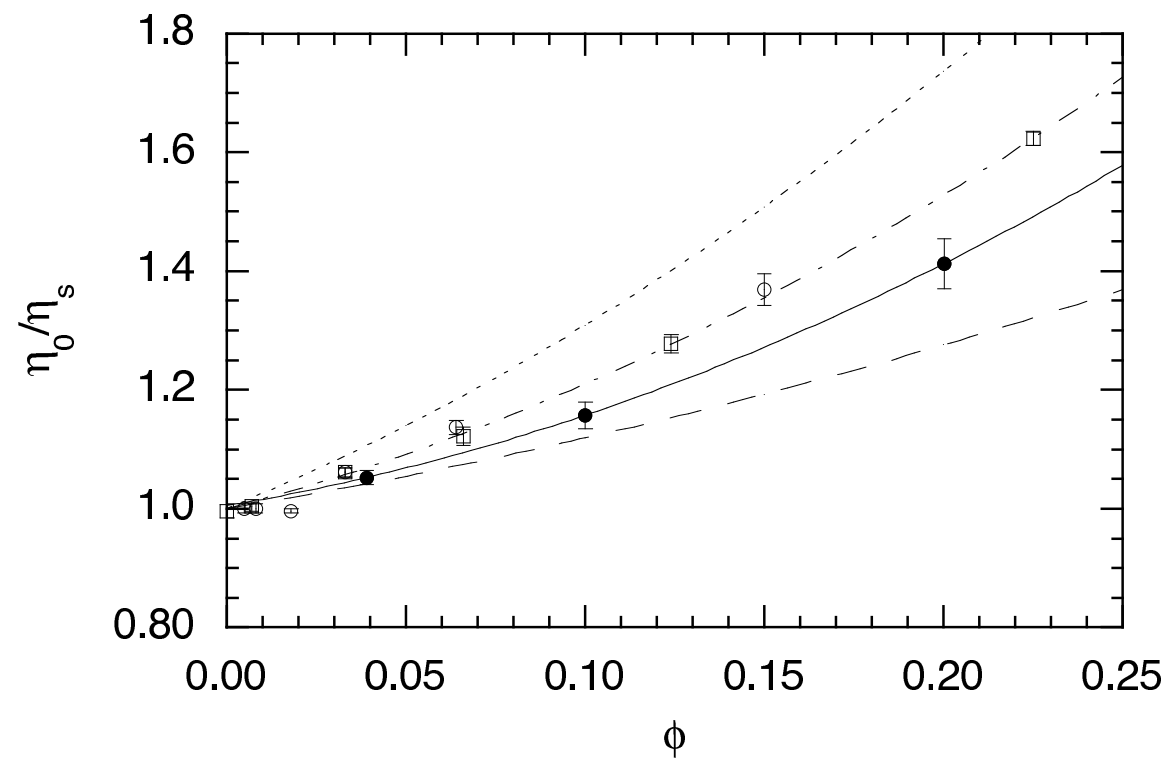

FIG. 8: Zero shear rate viscosities of the disparate-size mixtures plotted against the thermodynamic volume fraction. The unfilled circles are the results for the $x_{2}=0.005$ constant solute number fraction disparate-size mixtures and the squares are results for the $\mu=10$ constant mass ratio disparate-size mixtures. For comparison, the limiting infinite mass ratio viscosities from Fig. 4 are also shown (filled circles).

the constant $\mu=10$ data gives $\eta / \eta_{s}=1+1.6(2) \phi+5.4(8) \phi^{2}$ (shown as a dot-dash line in Fig. 8. The constant $x_{2}=0.005$ data covered a smaller range of volume fractions, and were better fitted by a straight line, with the result $\eta / \eta_{s}=1+1.2(3) \phi$. The fact that the disparate-size data and the extrapolated infinite mass ratio, $s=1$ data do not fall on a common curve when plotted against thermodynamic volume fraction indicates again that the thermodynamic volume fraction does not quite match the hydrodynamic volume fraction. The radius of the $\mu=10, s=2.225$ spheres calculated from the partial molecular volume is $r=1.25$, which can be compared with the interaction cross-radius of 1.61 and the bare radius 1.11 . For a mass ratio of $\mu=50$, we find that the radius from the partial molecular volume is 2.14 , the interaction cross-radius is 2.52 and the bare radius is 2.02 .

Using the same procedure as for the $s=1$ systems studied in the previous section, we can adjust the hydrodynamic radius to force the data to fit the theoretical curve for the viscosity of a hard sphere suspension with the slip boundary condition [34]. The results, shown in Fig. 9, show that a very good fit to the theoretical curve can be obtained by this procedure. The hydrodynamic radius used to calculate the volume fraction for this forced fit was given 
by $R_{H}=\left(c_{22}+1+\delta\right) / 2$ where the value of $\delta$ was adjusted to obtain an adequate fit. For the constant $\mu=1, s=2.225$ series of data, a value of $\delta=0.7$ was required, giving $R_{H}=1.46$. For the constant $x_{2}=0.005$ series, we found $\delta=0.2$. For the $\mu=50$ mass ratio, this gives a hydrodynamic radius of $R_{H}=2.11$.

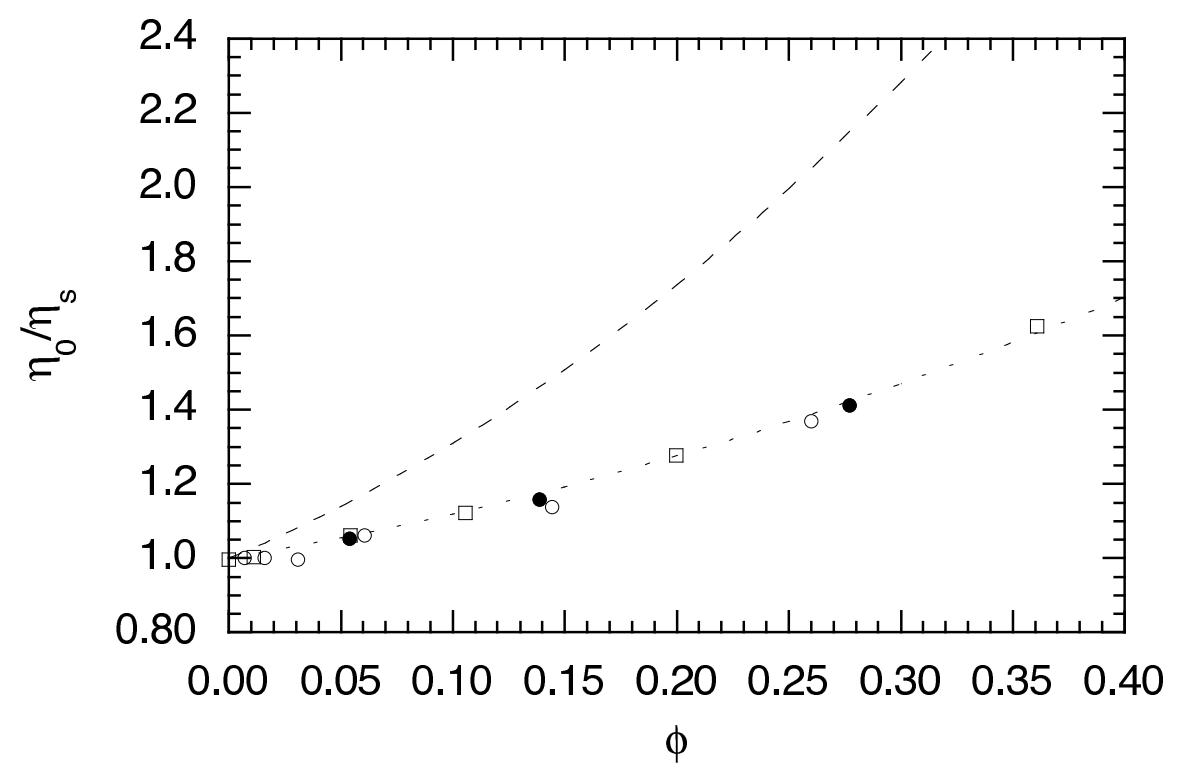

FIG. 9: Relative zero shear rate viscosity plotted against hydrodynamic volume fraction calculated with an adjustable parameter. The filled circles are the extrapolated infinite mass ratio, $s=1$ data, the unfilled circles are the results for the mixtures with constant number fraction $x_{2}=0.005$ and variable size and mass ratio, the squares for the constant mass ratio $\mu=10$, and size ratio $s=2.225$ mixtures with variable concentration. The short dashed line represents the theoretical result for a system of hydrodynamically interacting hard spheres with slip boundary condition and the long dashed line is the result for the stick boundary condition.

A summary of the results that we have obtained for the difference between the hydrodynamic radius and the bare particle radius of solute particles suspended in an explicitly simulated solvent is given in Table VIII.

\section{CONCLUSIONS}

Our simulations have shown that the viscosity of a suspension of small solute particles becomes strongly shear thinning when the solute to solvent mass ratio or size ratio is increased. Increasing the volume fraction of solute dramatically increases the rate of shear thinning 
TABLE VIII: Values of the difference between the hydrodynamic radius and the bare sphere radius, $\delta=R_{H}-s / 2$, calculated by various methods.

\begin{tabular}{ccccc}
\hline \hline$\mu$ & $s$ & $\delta($ cross-radius $)$ & $\delta(\eta(\phi))$ & $\delta$ (Stokes-Einstein) \\
\hline$\infty$ & 1 & 0.5 & 0.23 & 0.13 \\
10 & 2.225 & 0.5 & 0.35 & \\
50 & 4.03 & 0.5 & 0.1 & \\
\hline \hline
\end{tabular}

when the mass or size ratio is greater than one. For a series of systems in which the mass ratio is increased at constant size rate $s=1$, the zero shear rate viscosity approaches mass ratio independence at a rate that depends on the concentration. The rate of approach to mass independence surprisingly varies non-monotonically with concentration, unlike the rate of approach to mass independence of the self diffusion coefficient. The most rapid approach to mass ratio independence for the solution viscosity occurs at a solute number fraction of $x_{2}=0.1$. The asymptotic infinite mass ratio self diffusion coefficient extrapolated to zero concentration and the solvent viscosity can be used to obtain the hydrodynamic radius of the solute particles in the infinite mass ratio limit. When the slip boundary condition is assumed, we obtain a value for the hydrodynamic radius that agrees reasonably well with the value obtained by fitting the infinite mass ratio viscosity versus volume fraction data with an adjustable hydrodynamic radius to calculate the volume fraction. Both values are less than the cross-interaction radius between the solvent and solute molecules.

A similar set of simulations in which the size ratio was also varied produced results showing that the hydrodynamic radius was again larger than the bare spherical radius of the solute particles, but less than the cross-interaction radius.

In all of the above analysis, it has been assumed that the perfect slip hydrodynamic boundary condition is satisfied. In future work, better agreement between the different results would probably be obtained by independently determining the degree of slip and the hydrodynamic radius. 


\section{APPENDIX: ZHANG INTEGRATOR FOR A NOSÉ-HOOVER BAROSTAT}

The SLLOD equations of motion, with a Gaussian isokinetic thermostat and a NoséHoover barostat, equations (5), were used in this study. We also chose to use a symplectic integration scheme based on that developed by Zhang and Martyna [25] and [26, Appendix B]. This integration scheme requires the splitting of the equations of motion into parts which are exactly soluble, and then factorising the propagator to mimimise error. Here we detail the further steps required for the inclusion of the barostat.

With the inclusion of the barostat the positional equations of motion, and the associated split equations of motion $E_{1}$, become

$$
E_{1}=\left\{\begin{array}{l}
\dot{\mathbf{r}}_{i}=\frac{\mathbf{p}_{i}}{m_{i}}+\mathbf{i} \dot{\gamma} y_{i}-\zeta \mathbf{r}_{i} \\
\dot{\mathbf{p}}_{i}=0 \\
\dot{\zeta}=0 \\
\dot{V}=3 \zeta V
\end{array},\right.
$$

where the centre of mass $(\mathbf{R})$ is set to zero for simplicity, although it is not necessary. This set of equations has the exact solution

$$
\begin{aligned}
& x_{i}(t)=x(0) \mathrm{e}^{-\zeta t}+\left(\frac{p_{x i}}{m_{i}}+\dot{\gamma} \frac{p_{y i}}{m_{i}}\right) \frac{1}{\zeta}\left(1-\mathrm{e}^{-\zeta t}\right)+\dot{\gamma}\left(y(0)-\frac{1}{\zeta} \frac{p_{y i}}{m_{i}}\right) t \mathrm{e}^{-\zeta t} \\
& y_{i}(t)=y(0) \mathrm{e}^{-\zeta t}+\frac{p_{y i}}{m_{i}} \frac{1}{\zeta}\left(1-\mathrm{e}^{-\zeta t}\right) \\
& z_{i}(t)=z(0) \mathrm{e}^{-\zeta t}+\frac{p_{z i}}{m_{i}} \frac{1}{\zeta}\left(1-\mathrm{e}^{-\zeta t}\right)
\end{aligned}
$$

and

$$
\begin{aligned}
\mathbf{p}_{i}(t) & =\mathbf{p}_{i}(0) \\
\zeta(t) & =\zeta(0) \\
V(t) & =V(0) e^{3 \zeta t} .
\end{aligned}
$$

There are two sets of equations for the momenta equations of motion, a driven and undriven set, both of which are unchanged from the original work, [25] and [26, Appendix B]. That 
is,

$$
E_{2}=\left\{\begin{array}{l}
\dot{\mathbf{r}}_{i}=0 \\
\dot{\mathbf{p}}_{i}=\mathbf{F}_{i}-\alpha_{0} \mathbf{p}_{i}, \quad \alpha_{0}=\frac{1}{2 K_{0}} \sum_{i} \frac{\mathbf{p}_{i}}{m_{i}} \cdot \mathbf{F}_{i}, \\
\dot{\zeta}=0 \\
\dot{V}=0
\end{array}\right.
$$

and

$$
E_{3}=\left\{\begin{array}{l}
\dot{\mathbf{r}}_{i}=0 \\
\dot{\mathbf{p}}_{i}=-\mathbf{i} \dot{\gamma} p_{y i}-\alpha_{\gamma} \mathbf{p}_{i}, \quad \alpha_{\gamma}=-\frac{\dot{\gamma}}{2 K_{0}} \sum_{i} \frac{p_{x i} p_{y i}}{m_{i}}, \\
\dot{\zeta}=0 \\
\dot{V}=0 .
\end{array}\right.
$$

The solutions of these sets of equations of motion may be found in the references.

One further set of split equations of motion is required for the Nosé-Hoover barostat multiplier. This set is

$$
E_{4}=\left\{\begin{array}{l}
\dot{\mathbf{r}}_{i}=0 \\
\dot{\mathbf{p}}_{i}=0 \\
\dot{\zeta}=\frac{1}{\tau_{p}}\left(\frac{p}{p_{0}}-1\right) \\
\dot{V}=0
\end{array}\right.
$$

which has the simple solution $\mathbf{r}_{i}(t)=\mathbf{r}_{i}(0), \mathbf{p}_{i}(t)=\mathbf{p}_{i}(0), \zeta(t)=\zeta(0)+\frac{1}{\tau_{p}}\left(\frac{p}{p_{0}}-1\right) \times t$ and $V(t)=V(0)$. These equations of motion are then applied by using the factorizing the propagator using the Trotter scheme. That is,

$$
e^{i L \Delta t}=e^{E_{4} \Delta t / 2} e^{E_{3} \Delta t / 2} e^{E_{2} \Delta t / 2} \times e^{E_{1} \Delta t} \times e^{E_{2} \Delta t / 2} e^{E_{3} \Delta t / 2} e^{E_{4} \Delta t / 2}+O\left(\Delta t^{3}\right) .
$$

More elucidating details may be found in the references.

\section{ACKNOWLEDGMENTS}

We wish to thank the Victorian Partnership of Advanced Computing (VPAC) and the Australian Partnership for Advanced Computing (APAC) for generous grants of computer 
time.

[1] J.-P. Hansen and I. R. McDonald, Theory of Simple Liquids (Academic Press, London, 1986), 2nd ed.

[2] J. M. Deutch and I. Oppenheim, J. Chem . Phys. 54, 3547 (1971).

[3] S. Bhattacharyya and B. Bagchi, J. Chem. Phys. 106, 1757 (1997).

[4] M. J. Nuevo, J. J. Morales, and D. M. Heyes, Phys. Rev. E 58, 5845 (1998).

[5] F. Ould-Kaddour and D. Levesque, Phys. Rev. E 63, 011205(9) (2001).

[6] I. K. Snook, B. O’Malley, M. G. McPhie, and P. J. Daivis, J. Mol. Liq. 103-104, 405 (2003), in press.

[7] J. R. Schmidt and J. L. Skinner, J. Chem. Phys. 119, 8062 (2003).

[8] G. K. Batchelor, J. Fluid Mech. 131, 155 (1983).

[9] G. K. Batchelor, J. Fluid Mech. 137, 467 (1983).

[10] A. Einstein, Investigations on the theory of the Brownian movement (Dover, New York, 1956), edited by R. Fürth, translated by A. D. Cowper.

[11] L. Bocquet, J.-P. Hansen, and J. Piasecki, J. Stat. Phys. 76, 527 (1994).

[12] T. Kihara, Intermolecular forces (Wiley, New York, 1976).

[13] J. D. Weeks, D. Chandler, and H. C. Andersen, J. Chem. Phys. 54, 5237 (1971).

[14] S. Hess, M. Kröger, and H. Voigt, Physica A 250, 58 (1998).

[15] D. J. Evans and G. P. Morriss, Statistical Mechanics of Nonequilibrium Liquids (Academic Press, New York, 1990).

[16] G. C. S. Melchionna and B. L. Holian, Mol. Phys. 78, 533 (1993).

[17] A. W. Lees and S. F. Edwards, J. Phys. C 5, 1921 (1972).

[18] M. Suzuki, Phys. Lett. A 165, 387 (1992).

[19] G. J. Martyna, D. J. Tobias, and M. L. Klein, J. Chem. Phys. 101, 4177 (1994).

[20] G. J. Martyna and M. E. Tuckerman, J. Chem. Phys. 102, 8071 (1995).

[21] D. J. Isbister, D. J. Searles, and D. J. Evans, Physica A 240, 105 (1997).

[22] D. J. Searles, D. J. Isbister, and D. J. Evans, Math. Comput. Simul. 45, 147 (1998).

[23] M. P. Allen and D. J. Tildesley, Computer simulation of liquids (Clarendon Press, Oxford, 1987). 
[24] W. C. Swoop, H. C. Andersen, P. H. Berens, and K. R. Wilson, J. Chem. Phys. 76, 637 (1982).

[25] F. Zhang, J. Chem. Phys. 106, 6102 (1997).

[26] F. Zhang, D. J. Searles, D. J. Evans, J. S. den Toom Hansen, and D. J. Isbister, J. Chem. Phys. 111, 18 (1999).

[27] D. J. Evans and G. P. Morriss, Comp. Phys. Rep. 1, 297 (1984).

[28] T. J. Murphy and J. L. Aguirre, J. Chem. Phys. 57, 2098 (1972).

[29] T. Yamada and K. Kawasaki, Prog. Theor. Phys. 53, 1111 (1973).

[30] D. J. Evans, G. P. Morriss, and L. M. Hood, Mol. Phys. 68, 637 (1989).

[31] K. P. Travis, D. J. Searles, and D. J. Evans, Mol. Phys. 95, 195 (1998).

[32] G. Marcelli, B. D. Todd, and R. J. Sadus, Phys. Rev. E 63, 021204(10) (2002).

[33] I. Borzsák, P. T. Cummings, and D. J. Evans, Mol. Phys. 100, 2735 (2002).

[34] E. Wajnryb and J. S. Dahler, Physica A 253, 77 (1998).

[35] R. I. Tanner, Engineering Rheology, vol. 52 of Oxford Engineering Science Series (Oxford University Press, Oxford, 2000), 2nd ed.

[36] R. G. Larson, The structure and rheology of complex fluids, Topics in complex engineering (Oxford University Press, New York, 1999).

[37] G. K. Batchelor, J. Fluid Mech. 83, 97 (1977).

[38] D. M. Heyes, Phys. Lett. A 132, 399 (1988). 